\title{
Geometric band properties in strained monolayer transition metal dichalcogenides using simple band structures
}

Cite as: J. Appl. Phys. 126, 115701 (2019); doi: 10.1063/1.5115093

Submitted: 14 June 2019 . Accepted: 24 August 2019.

Published Online: 17 September 2019

Shahnaz Aas (iD) and Ceyhun Bulutay ${ }^{a)}$ (iD

\author{
AFFILIATIONS \\ Department of Physics, Bilkent University, 06800 Bilkent, Ankara, Turkey
}

a)Electronic mail: bulutay@bilkent.edu.tr

\begin{abstract}
Monolayer transition metal dichalcogenides (TMDs) bare large Berry curvature hotspots readily exploitable for geometric band effects. Tailoring and enhancement of these features via strain is an active research direction. Here, we consider spinless two- and three-band and spinful four-band models capable to quantify the Berry curvature and the orbital magnetic moment of strained TMDs. First, we provide a $k \cdot p$ parameter set for $\mathrm{MoS}_{2}, \mathrm{MoSe}_{2}, \mathrm{WS}_{2}$, and $\mathrm{WSe}_{2}$ in the light of the recently released ab initio and experimental band properties. Its validity range extends from the $K$ valley edge to about one hundred millielectron volts into valence and conduction bands for these TMDs. To expand this over a larger part of the Brillouin zone, we incorporate strain to an available three-band tight-binding Hamiltonian. With these techniques, we demonstrate that both the Berry curvature and the orbital magnetic moment can be doubled compared to their intrinsic values by applying typically a $2.5 \%$ biaxial tensile strain. These simple band structure tools can find application in the quantitative device modeling of the geometric band effects in strained monolayer TMDs.
\end{abstract}

Published under license by AIP Publishing. https://doi.org/10.1063/1.5115093

\section{INTRODUCTION}

The monolayer transition metal dichalcogenides (TMDs) of the semiconducting $2 \mathrm{H}$ polytype avail a wide range of electrical, magnetic, optical, and mechanical control and tunability. ${ }^{1-3}$ Their valley-contrasting properties associated with the so-called inequivalent $K$ valleys at the corners of the hexagonal Brillouin zone grant information carriers the opportunity to experience nondissipative electronics. ${ }^{4}$ Unlike the similar multiple conduction band valleys in conventional bulk silicon electronics, in TMDs the valley degree of freedom is practically an individually accessible quantum label. ${ }^{5}$ For instance, in the so-called valley Hall effect, an in-plane electric field initiates a valley current in the transverse in-plane direction, ${ }^{6-8}$ which has been confirmed by both optical ${ }^{9}$ and transport $^{10}$ measurements.

At the heart of these valley-based physics, there lies the sublattice-driven orbital angular momentum. ${ }^{7,11}$ Alternatively, from the perspective of quantum geometrical band properties, ${ }^{12}$ the foregoing effects can be attributed to the Berry curvature (BC) and orbital magnetic moment (OMM).$^{13}$ Both of them take part in various phenomena such as the dichroic selection rules in optical absorption $^{14,15}$ or the excitonic $p$ level energy splitting which is proportional to the $\mathrm{BC}$ flux; ${ }^{16,17}$ OMM accounts for the interatomic currents (self-rotating motion of the electron wavepacket) ${ }^{18}$ responsible for the valley $g$-factor in TMDs. ${ }^{19,20}$ Thus, by breaking timereversal symmetry with a perpendicular magnetic field, a valley Zeeman splitting is introduced in addition to the well-known spin Zeeman effect. ${ }^{21,22}$ Very recently, through their intimate connection with the orbital angular momentum, these geometric band properties are locally mapped in momentum space using circular dichroism angle-resolved photoelectron spectroscopy.

A unique advantage of TMDs is their mechanical deformability up to at least $10 \%$ in their lattice constants without degradation. ${ }^{24}$ Undoubtedly, it is bound to have ramifications on the quantum geometric band properties, where a quantification inevitably necessitates band structure tools reliable under strain. The $k \cdot p$ method has been the first resort because of its simplicity, starting with graphene ${ }^{6}$ and carried over to other two-dimensional materials. ${ }^{20,25-28}$ Very recently a strained parametrization is also offered, ${ }^{29}$ which we used to successfully explain the experimental photoluminescence peak shifts in strained TMDs. ${ }^{30}$ On the other hand, it has a number of 
shortcomings especially for studying carrier transport away from the $K$ point. For example, it is isotropic, preserves the electron-hole symmetry, and remains parabolic. In contrast, TMDs possess the trigonal warping (TW) of the isoenergy contours which leads to measurable effects in the polarization of electroluminescence in $p-n$ junctions. $^{31}$ The electron-hole symmetry breaking has been confirmed by magnetoluminescence experiments. ${ }^{32,33}$ Lastly, the bands quickly display nonparabolic dependence away from the valley minimum ${ }^{34}$ which among other quantities directly affects the BC and $\mathrm{OMM}^{35}$

Another prevailing band structure choice is the tight-binding model for which a number of parametrizations exist for monolayer TMDs. ${ }^{2,29,36-39}$ Compared to $k \cdot p$, their agreement with first-principles data is over a much wider range of the Brillouin zone, which comes at a price of some added formulation complexity and larger number of fitting parameters. Among these, arguably the simplest to use is the one by Liu et al., which is unfortunately only available for unstrained TMDs. ${ }^{38}$ It should be noted that both $k \cdot p$ and tight-binding models warrant analytically tractable transparent physics. In the literature, there is also a vast amount of density functional theory (DFT) based results ${ }^{20,29,40,41}$ which are highly reliable, other than the well known underestimation of the bandgap by most DFT exchange-correlation functionals. ${ }^{42}$ This entails further techniques like the many-body $G W$ approximation, which yields bandgaps much closer to experiments, albeit being computationally very demanding, and so far practically inapplicable to systems beyond a few tens of atoms in the unit cell, ${ }^{43}$ making them highly undesirable for device modeling purposes.

The aim of this work is to present simple band structure options that can quantify the changes under strain in the $\mathrm{BC}$ and the OMM around a wider portion of the $K$ valleys. For this purpose, to alleviate the drawbacks of existing strained $k \cdot p$ parametrization, such as disagreement with the reported electron and hole effective masses as well as the bandgap values, ${ }^{29}$ we develop two-band spinless and four-band spinful versions taking into account up-to-date first-principles and experimental data including quantum geometrical band properties, as will be described below. The agreement window with the $a b$ initio and tight-binding band structures falls in the range $70-400 \mathrm{meV}$ from the $K$ valley edge for the TMDs targeted in this work: $\mathrm{MoS}_{2}, \mathrm{MoSe}_{2}, \mathrm{WS}_{2}$, and $\mathrm{WSe}_{2}$. Moreover, we extend the tight-binding approach by Liu et al..$^{38}$ to uniaxial and biaxial strain conditions. Based on these tools, we demonstrate a doubling of $\mathrm{BC}$ and OMM for both the valence band (VB) and the conduction band (CB) under about 2.5\% tensile biaxial strain. We also present a simple explanation of how strain modifies these quantum geometrical band properties.

\section{THEORY}

\section{A. Two-band $\boldsymbol{k} \cdot \boldsymbol{p}$ Hamiltonian}

For carriers near the $K$ valley edges of monolayer TMDs, the two-band $k \cdot p$ low-energy Hamiltonian $\left(H_{0}\right)$ which is dominated by the metal atom's open $d$ shell orbitals is the starting point of many studies. ${ }^{8}$ In the presence of strain, characterized by the tensor components $\varepsilon_{i j}$ such that $\{i, j\} \in\{x, y\}$, an extra term $\left(H_{\varepsilon}\right)$ is introduced. $^{29}$ These two Hamiltonians are described in the Bloch basis

$$
\begin{gathered}
\text { of }\left\{\left|\boldsymbol{k}, d_{z^{2}}\right\rangle,\left|\boldsymbol{k}, d_{x^{2}-y^{2}}+i d_{x y}\right\rangle\right\} \text { by } \\
H_{0}=\frac{f_{1}}{2} \sigma_{z}+f_{2} a\left(k_{x} \sigma_{x}+k_{y} \sigma_{y}\right), \\
H_{\varepsilon}=f_{4}\left(\varepsilon_{x x}+\varepsilon_{y y}\right) \sigma_{z}+f_{5}\left[\left(\varepsilon_{x x}-\varepsilon_{y y}\right) \sigma_{x}-2 \varepsilon_{x y} \sigma_{y}\right],
\end{gathered}
$$

where $k_{i}$ is the wave vector Cartesian component centered around the corresponding $K$ point, $f$ 's are the fitted parameters for different TMD materials, $a$ is the lattice constant, and $\sigma_{i}$ 's are the Pauli matrix Cartesian components. The expressions in this subsection specifically apply for the $+K$ valley, while those for the $-K$ valley can be obtained by the complex conjugation of the matrix entries. ${ }^{34}$ Also, we drop the constant midgap position parameters $f_{0}$ and $f_{3}$ in Ref. 29, which need to be reinstated in the study of heterostructures for their proper band alignment.

To account for additional features of electron-hole asymmetry, TW, and nonparabolicity, we follow Kormányos et al. ${ }^{34}$ by including three more terms

$$
H_{2 \mathrm{~B}}(\boldsymbol{k})=H_{0}+H_{\varepsilon}+H_{\mathrm{asym}}+H_{\mathrm{TW}}+H_{\text {cubic }}
$$

where

$$
\begin{gathered}
H_{\text {asym }}=\left(\begin{array}{cc}
\beta k^{2} & 0 \\
0 & \alpha k^{2}
\end{array}\right), \\
H_{\mathrm{TW}}=\kappa\left(\begin{array}{cc}
0 & k_{+}^{2} \\
k_{-}^{2} & 0
\end{array}\right), \\
H_{\text {cubic }}=\frac{\eta}{2} k^{2}\left(\begin{array}{cc}
0 & k_{-} \\
k_{+} & 0
\end{array}\right),
\end{gathered}
$$

and $k_{ \pm}=k_{x} \pm i k_{y}$, where the parameters $\alpha$ and $\beta$ describe the breaking of the electron-hole symmetry, whereas $\kappa$ is responsible for the TW of the isoenergy contours, and $H_{\text {cubic }}$ serves to improve the fit further away from the $K$ point. ${ }^{34}$

\section{B. Three-band tight-binding Hamiltonian}

The two-band $k \cdot p$ approach is inevitably restricted to the vicinity of the $K$ points. To extend it over a wider part of the Brillouin zone, the number of bands needs to be increased considerably. ${ }^{20}$ For the sake of simplicity, we rather prefer the three-band tight-binding (TB) approach which provides a full-zone band structure fitted to the first-principles data, where in the case of up to third nearest neighbor interactions 19 fitting parameters are involved. ${ }^{38}$ It assumes the Bloch basis of $\left\{\left|\boldsymbol{k}, d_{z^{2}}\right\rangle,\left|\boldsymbol{k}, d_{x y}\right\rangle,\left|\boldsymbol{k}, d_{x^{2}-y^{2}}\right\rangle\right\}$ coming from the atomic orbitals that largely contribute to the VB and CB edges of TMDs. ${ }^{34}$ The matrix representation of the Hamiltonian takes the form

$$
H_{0}=\left(\begin{array}{ccc}
V_{0} & V_{1} & V_{2} \\
V_{1}^{*} & V_{11} & V_{12} \\
V_{2}^{*} & V_{12}^{*} & V_{22}
\end{array}\right),
$$


where $V_{0}, V_{1}, V_{2}, V_{11}, V_{12}$, and $V_{22}$ are the TB matrix elements; for their detailed expressions, we refer to Ref. 38. Though this Hamiltonian is highly satisfactory, it is for unstrained TMDs. We remedy this by the two-band deformation potentials proposed by Fang et al. ${ }^{29}$ that we also use in our $k \cdot p$ theory in Sec. II A. So, the strain is embodied into the three-band TB Hamiltonian as

$$
H_{\varepsilon}=\left(\begin{array}{ccc}
e_{a} & e_{b} & e_{b} \\
e_{b} & -e_{a} & 0 \\
e_{b} & 0 & -e_{a}
\end{array}\right),
$$

where

$$
\begin{aligned}
& e_{a}=f_{4}\left(\varepsilon_{x x}+\varepsilon_{y y}\right), \\
& e_{b}=f_{5}\left(\varepsilon_{x x}-\varepsilon_{y y}\right) .
\end{aligned}
$$

Here, our simplistic approach lends itself to a number of restrictions. Even though this TB is a three-band model, the deformation potentials are only available for the two-band case (highest VB and the lowest CB). ${ }^{29}$ Therefore, we expand it to the two-dimensional subspace formed by $\left|\boldsymbol{k}, d_{x y}\right\rangle$ and $\left|\boldsymbol{k}, d_{x^{2}-y^{2}}\right\rangle$, which define the highest VB and the first-excited $\mathrm{CB}$ around the $K$ valleys, while neglecting the strain coupling between them. Its form [Eq. (8)] complies with the TB $d-d$ sector deformation coupling of monolayer TMDs. ${ }^{27}$ As another remark, here strain only acts through the uniaxial and biaxial components, with no involvement of the shear strain $\left(\varepsilon_{x y}=\varepsilon_{y x}\right)$. In fact, it has been shown for this level of theory that the latter is only responsible for a rigid shift of the band extrema. ${ }^{29,30}$

To test the validity of this simple strain extension, in Fig. 1, we compare it with the first-principles band structure results for $\mathrm{WSe}_{2}$ under $\pm 2 \%$ biaxial and unstrained cases. ${ }^{29}$ As intended, the agreement around the $K$ valley is quite satisfactory, whereas disagreement sets in away from this region especially toward the $\Gamma$ point. Apparently, $\Gamma$ and $K$ valleys have different signs for the deformation potentials causing a direct to indirect transition under compressive strain. Thus, it cannot be represented with only that of a single (i.e., $K$ ) valley. As a matter of fact, even for the unstrained case, the original TB fitting has deficiencies around the $\Gamma$ point. ${ }^{38}$ These limitations will not be of practical concern for this work as the geometrical band properties that we are interested in are localized around the $K$ point and vanish toward the $\Gamma$ point due to symmetry considerations. ${ }^{44}$

\section{Berry curvature and orbital magnetic moment}

In the absence of an external magnetic field, TMDs respect the time reversal symmetry, but inversion symmetry is broken in monolayers or odd number of layers as has been independently confirmed by recent experiments. ${ }^{9,10}$ Therefore, in monolayer TMDs, BC has a nonzero value with opposite sign in $K$ and $-K$ valleys connected by a time-reversal operation. ${ }^{13}$ For a chosen band with label $n$, it can be calculated without reference to other bands using

$$
\Omega_{n, z}(\boldsymbol{k})=-2 \operatorname{Im}\left\langle\partial_{k_{x}} u_{n \boldsymbol{k}} \mid \partial_{k_{y}} u_{n \boldsymbol{k}}\right\rangle,
$$

where $z$ is the direction perpendicular to monolayer plane and $\left|u_{n k}\right\rangle$ is the cell-periodic part of the Bloch function at wave vector $\boldsymbol{k}^{12}$ Another geometric band property is the OMM which is also a pseudovector given by

$$
\mu_{n, z}(\boldsymbol{k})=2 \frac{\mu_{B} m_{0}}{\hbar^{2}} \operatorname{Im}\left\langle\partial_{k_{x}} u_{n \boldsymbol{k}}\left|\left[H(\boldsymbol{k})-E_{n}(\boldsymbol{k})\right]\right| \partial_{k_{y}} u_{n \boldsymbol{k}}\right\rangle
$$

where $\mu_{B}$ is the Bohr magneton, $m_{0}$ is the free-electron mass, and $E_{n}(\boldsymbol{k})$ is the energy of the band $n$ at the wave vector $\boldsymbol{k}$.

\section{Fitting procedure and data references}

Our two-band $k \cdot p$ model depends on the following parameters: $a, f_{1}, f_{2}, f_{4}, f_{5}, \alpha, \beta, \kappa$, and $\eta$. The lattice constant, $a$, is taken from DFT (GGA) model calculations (Table I). ${ }^{38}$ For the remaining eight parameters, rather than going through a formidable simultaneous optimization in such a high-dimensional parameter space, a sequential fitting is possible as follows. $f_{1}$ determines the free-
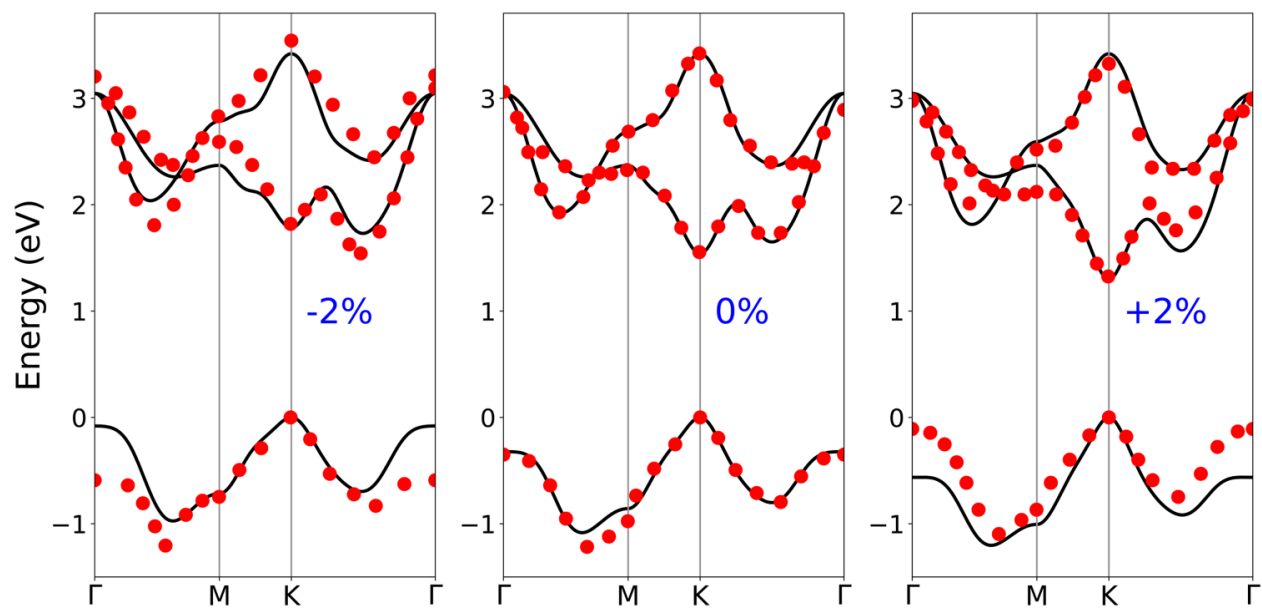

FIG. 1. Comparison of first-principles band structure (red dots) $)^{29}$ with the TB results (black line) for $\mathrm{WSe}_{2}$ under unstrained $(0 \%)$ and $\pm 2 \%$ biaxial strain cases. Energy reference is set to VB maximum for each case. 
TABLE I. Target data for $k \cdot p$ parameters. Lattice constant $(a),{ }^{38}$ single-particle bandgap $\left(E_{g}\right),{ }^{45}$ average of $\mathrm{BC}(\bar{\Omega}),{ }^{44} \mathrm{VB}\left(m_{\mathrm{vb}}^{*}\right)$, and CB $\left(m_{\mathrm{cb}}^{*}\right)$ effective masses. ${ }^{25}$ $E_{g}$ and the two-band $k \cdot p$ parameters are based on spin-allowed lowest energy VB-CB transition between spin-down $(\downarrow)$ states according to Ref. 25 .

\begin{tabular}{lcccc}
\hline \hline Materials & $\mathrm{MoS}_{2}$ & $\mathrm{MoSe}_{2}$ & $\mathrm{WS}_{2}$ & $\mathrm{WSe}_{2}$ \\
\hline$a(\AA)$ & 3.190 & 3.326 & 3.191 & 3.325 \\
$E_{g}(\mathrm{eV})$ & 2.15 & 2.18 & 2.38 & 2.20 \\
$|\Omega|\left(\AA^{2}\right)$ & 10.43 & 10.71 & 16.03 & 17.29 \\
$m_{\mathrm{vb}, \downarrow}^{*}\left(m_{0}\right)$ & -0.54 & -0.59 & -0.35 & -0.36 \\
$m_{\mathrm{cb}, \downarrow}\left(m_{0}\right)$ & 0.43 & 0.49 & 0.26 & 0.28 \\
$m_{\mathrm{vb}, \uparrow}^{*}\left(m_{0}\right)$ & -0.61 & -0.7 & -0.49 & -0.54 \\
$m_{\mathrm{cb}, \uparrow}^{*}\left(m_{0}\right)$ & 0.46 & 0.56 & 0.35 & 0.39 \\
\hline \hline
\end{tabular}

particle bandgap which we fit to the corresponding experimental $E_{g}$ using scanning tunneling spectroscopy data (i.e., without the excitonic contributions) listed in the recent review (Table I). ${ }^{45}$ For $f_{2}$, we make use of the fact that the $\mathrm{BC}$ expression at the $K$ point simplifies to $\Omega(K)= \pm 2\left(f_{2} a / f_{1}\right)^{2}$. ${ }^{6}$ We fit the average of this quantity for the lowest spin-allowed transitions in $K$ valley $\left(|\bar{\Omega}|=\left(\left|\Omega_{\mathrm{CB}}(K)\right|+\left|\Omega_{\mathrm{VB}}(K)\right|\right) / 2\right)$ to the first-principles results (Table I) ${ }^{44}$ which resolves the $f_{2}$ parameter. $f_{4}$ and $f_{5}$ characterize the strain and they are directly acquired from Ref. 29 without any change. After these set of parameters for $H_{0}$, we move to $H_{\text {asym }}$ for $\alpha$ and $\beta$. We readily extract these from the reported effective masses (Table I). ${ }^{25}$ As a two-band model, again we select the effective masses of lowest spin-allowed VB-CB transitions in the fitting procedure.

Figure 2 compares the isoenergy contours plotted using Eqs. (1) and (4) (solid lines), with the TB model calculations ${ }^{38}$ (dashed lines). Each color corresponds to a different amount of excess energy as measured from the VB $K$ valley edge (i.e., VB maximum). Figure 2(a) displays the case without TW in $k \cdot p$ calculations resulting in circular curves. By adding Eq. (5) to the previous Hamiltonian [Eqs. (1) and (4)], the TW effect on the isoenergy contours emerges [Fig. 2(b)]. We fix the $\kappa$ parameter by fitting the $k \cdot p$ to the TB model at the $100 \mathrm{meV}$ isoenergy contour. Finally, to extract the $\eta$ parameter, we fit the band structure of different TMDs calculated from Eq. (3) to the recent DFT data. Our final two-band $k \cdot p$ parameter set for the four TMDs is presented in Table II.

Figure 3 contrasts the band structure of different TMDs from Eq. (1) (red curves), and including additional terms in Eq. (3) using our $k \cdot p$ fitted parameters (blue curves) along with the DFT values (yellow dots). ${ }^{20,29,40,41}$ Furthermore, we plot TB band structures $^{38}$ (black dashed curves) in this figure to assess how precise is our two-band model. Notably, DFT and TB model are in excellent agreement. Also, the blue curves from the two-band $k \cdot p$ Hamiltonian, $H_{2 B}$ calculations approach to DFT and TB model results around the $K$ valley which assure the benefit of these additional terms [Eqs. (4)-(6)] in Eq. (1). Within $10 \mathrm{meV}$ agreement window with TB and DFT data ${ }^{20,29,40,41}$ is included in Table II. The narrowest among these is for $\mathrm{WS}_{2} \mathrm{CB}$ which is $70 \mathrm{meV}$ and widest for $\mathrm{MoSe}_{2}$ for VB with $400 \mathrm{meV}$, both as measured from the respective band edges. Thus, for intravalley transport, these can suffice, except for the hot carrier regime for which we advise to switch to the TB model.

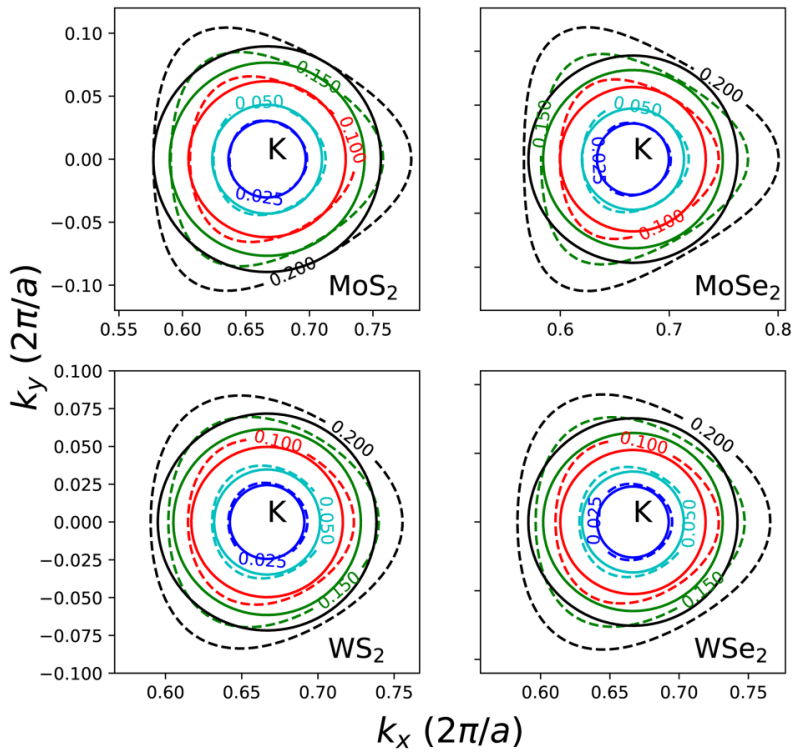

(a)

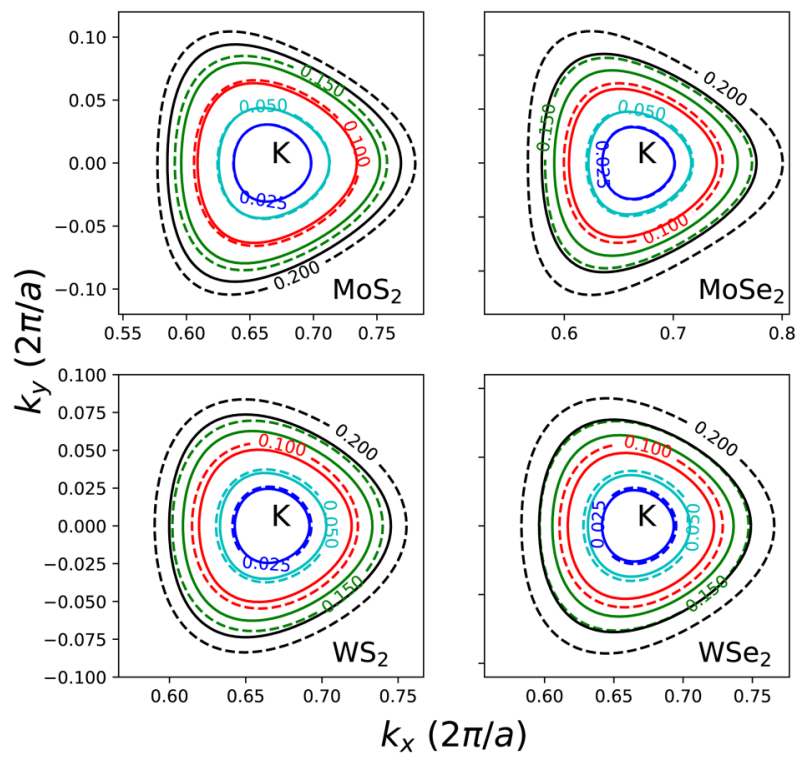

(b)

FIG. 2. Isoenergy contours of the VB (at energies indicated in electronvolts) for different TMDs from TB (dashed) and $k \cdot p$ (solid) models, where the latter excludes (a) or includes (b) the TW effect. For these plots, the $\Gamma$ point has been taken as the origin for $\boldsymbol{k}$.

\section{E. Spin-dependent four-band $\boldsymbol{k} \cdot \boldsymbol{p}$ Hamiltonian}

Due to the presence of heavy metal atoms in TMDs, the spin-orbit interaction is quite strong ${ }^{27}$ in contrast to, for instance, monolayer graphene and $\mathrm{hBN} .^{29}$ The spin-dependent effects in 
TABLE II. Fitted two-band $k \cdot p$ parameters and within $10 \mathrm{meV}$ agreement window for $V B$ and $C B$.

\begin{tabular}{lcccc}
\hline \hline TMD & $\mathrm{MoS}_{2}$ & $\mathrm{MoSe}_{2}$ & $\mathrm{WS}_{2}$ & $\mathrm{WSe}_{2}$ \\
\hline$f_{1}(\mathrm{eV})$ & 2.15 & 2.18 & 2.38 & 2.2 \\
$f_{2}(\mathrm{eV})$ & 1.54 & 1.52 & 2.11 & 1.95 \\
$f_{4}(\mathrm{eV})$ & -2.59 & -2.28 & -3.59 & -3.02 \\
$f_{5}(\mathrm{eV})$ & 2.2 & 1.84 & 2.27 & 2.03 \\
$\alpha\left(\mathrm{eV} \AA^{2}\right)$ & 4.16 & 5.22 & 8.2 & 8.43 \\
$\beta\left(\mathrm{eV}^{2}\right)$ & -2.35 & -3.9 & -4.43 & -5.4 \\
$\kappa\left(\mathrm{eV} \AA^{2}\right)$ & -1.9 & -1.8 & -2.2 & -2 \\
$\eta\left(\mathrm{eV} \AA^{3}\right)$ & 6 & 8 & 14 & 18 \\
VB fit range $(\mathrm{meV})$ & 350 & 400 & 200 & 100 \\
$\mathrm{CB} \mathrm{fit} \mathrm{range}(\mathrm{meV})$ & 115 & 170 & 70 & 90 \\
\hline \hline
\end{tabular}

TMDs are commonly incorporated within the spin-diagonal and wave vector-independent approximation. ${ }^{8,25,34,38}$ Thus, we first generalize the two-band Hamiltonian of Eq. (3) into a form with spindependent diagonal entries as

$$
H_{2 \mathrm{~B}}=\left(\begin{array}{ll}
h_{11} & h_{12} \\
h_{12}^{*} & h_{22}
\end{array}\right) \longrightarrow H_{2 \mathrm{~B}}^{\downarrow, \uparrow}=\left(\begin{array}{ll}
h_{11}^{\downarrow, \uparrow} & h_{12} \\
h_{12}^{*} & h_{22}^{\downarrow, \uparrow}
\end{array}\right)
$$

by modifying only the electron-hole asymmetry contribution in

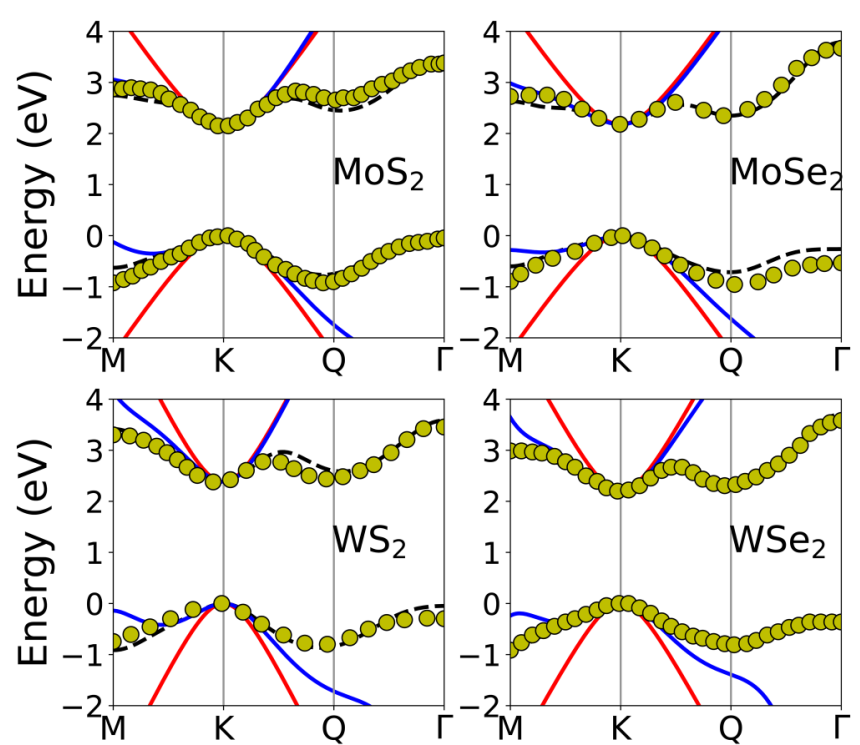

FIG. 3. $k \cdot p$ band structure of monolayer TMDs with (blue) and without (red) taking into account the electron-hole asymmetry, TW, and nonparabolic effects, compared with the TB calculations (black dashed lines) and DFT results (yellow dots) collected from Refs. 20, 29, 40, and 41. To facilitate the comparison, VB maxima are set to zero energy, and the bandgaps in each case are corrected to the values in Table I.
TABLE III. Additional spin-dependent $k \cdot p$ parameters required for the four-band Hamiltonian. CB and VB spin splittings are taken from spin-polarized DFT band structure. ${ }^{25} \alpha^{\downarrow}$ and $\beta^{\downarrow}$ values coincide with the two-band values in Table II.

\begin{tabular}{lcccc}
\hline \hline $\mathrm{TMD}$ & $\mathrm{MoS}_{2}$ & $\mathrm{MoSe}_{2}$ & $\mathrm{WS}_{2}$ & $\mathrm{WSe}_{2}$ \\
\hline$\Delta_{\mathrm{cb}}^{\mathrm{so}}(\mathrm{meV})$ & -3 & -22 & 32 & 37 \\
$\Delta_{\mathrm{vb}}^{\mathrm{so}}(\mathrm{meV})$ & 148 & 186 & 429 & 466 \\
$\alpha^{\downarrow}\left(\mathrm{eV} \mathrm{\AA}^{2}\right)$ & 4.16 & 5.22 & 8.2 & 8.43 \\
$\beta^{\downarrow}\left(\mathrm{eV} \AA^{2}\right)$ & -2.35 & -3.9 & -4.43 & -5.4 \\
$\alpha^{\uparrow}\left(\mathrm{eV} \AA^{2}\right)$ & 4.23 & 5.22 & 8.58 & 8.85 \\
$\beta^{\uparrow}\left(\mathrm{eV} \AA^{2}\right)$ & -2.2 & -3.86 & -5.47 & -6.15 \\
\hline \hline
\end{tabular}

Eq. (4) so that it becomes

$$
H_{\mathrm{asym}}^{\downarrow, \uparrow}=k^{2}\left(\begin{array}{cc}
\beta^{\downarrow, \uparrow} & 0 \\
0 & \alpha^{\downarrow, \uparrow}
\end{array}\right),
$$

where the fitted $\beta^{\downarrow, \uparrow}$ and $\alpha^{\downarrow, \uparrow}$ to the corresponding effective mass values are tabulated in Table III. We keep the remaining two-band $k \cdot p$ parameters $\left(f_{1}, f_{2}, f_{4}, f_{5}, \kappa, \eta\right)$ as in Table II, and in this way, we do not inflate the number of fitting parameters significantly.

With these ingredients the two-band formalism is extended into both spin channels that results in the four-band $k \cdot p$ Hamiltonian which is expressed in the Bloch basis ordering of $\left\{\left|\boldsymbol{k}, d_{z^{2}}, \uparrow\right\rangle,\left|\boldsymbol{k}, d_{z^{2}}, \downarrow\right\rangle,\left|\boldsymbol{k}, d_{x^{2}-y^{2}}+i d_{x y}, \uparrow\right\rangle,\left|\boldsymbol{k}, d_{x^{2}-y^{2}}+i d_{x y}, \downarrow\right\rangle\right\}$ as

$$
\begin{aligned}
H_{4 \mathrm{~B}}= & \left(\begin{array}{cccc}
h_{11}^{\uparrow} & 0 & h_{12} & 0 \\
0 & h_{11}^{\downarrow} & 0 & h_{12} \\
h_{12}^{*} & 0 & h_{22}^{\uparrow} & 0 \\
0 & h_{12}^{*} & 0 & h_{22}^{\downarrow}
\end{array}\right) \\
& +\tau\left(\begin{array}{cccc}
-\Delta_{\mathrm{cb}}^{\mathrm{so}} & 0 & 0 & 0 \\
0 & 0 & 0 & 0 \\
0 & 0 & -\Delta_{\mathrm{vb}}^{\mathrm{so}} & 0 \\
0 & 0 & 0 & 0
\end{array}\right),
\end{aligned}
$$

where $\tau$ is the valley index with value $+1(-1)$ for the $+K(-K)$ valley, and $\Delta_{\mathrm{cb}}^{\mathrm{so}}\left(\Delta_{\mathrm{vb}}^{\mathrm{so}}\right)$ is the $\mathrm{CB}(\mathrm{VB})$ spin splitting as listed in Table III.

Figure 4 shows the spin-dependent band structure of the monolayer TMDs around the $K$ valley. As the spin-dependent parameters in Eq. (15) reside on the diagonal entries, in this level of approximation spin remains to be a good quantum label. ${ }^{8}$ Another convenience of this approach is that the aforementioned two-band model directly corresponds to the spin- $\downarrow$ sector of the four-band Hamiltonian. Therefore, when lowest-lying spin-allowed transitions (as in the so-called $A$-excitons) are of interest, ${ }^{45}$ the spinless two-band variant in Sec. II A can be employed.

\section{RESULTS AND DISCUSSION}

To demonstrate several aspects of the geometric band properties, we choose monolayer $\mathrm{WSe}_{2}$ as the prototypical TMD material 

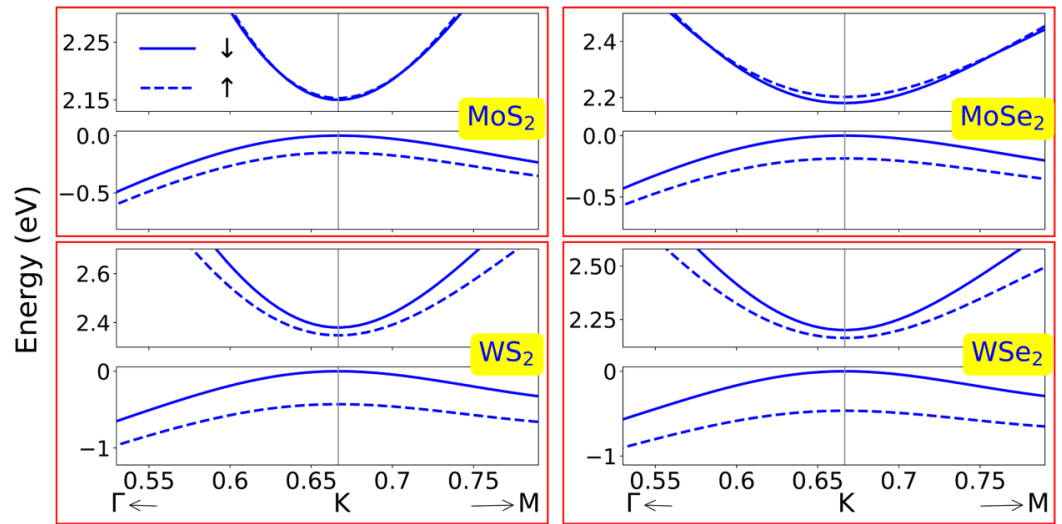

FIG. 4. Four-band $k \cdot p$ band structure of monolayer TMDs around the $K$ valley for spin $\downarrow$ (solid) and spin $\uparrow$ (dashed) bands. Abscissas are in units of $2 \pi / a$. Spin-dependent parameters are listed in Table III, and the remaining spin-independent parameters $\left(f_{1}, f_{2}, f_{4}, f_{5}, \kappa, \eta\right)$ are used from Table II.

and focus on the bands with the narrowest spin-allowed bandgap transition which corresponds to spin- $\downarrow$ sector at the $K$ valley (solid lines in Fig. 4) which essentially reduces the computational task to the two-band $k \cdot p$, and the three-band TB cases, as mentioned above. Starting with the unstrained case in Fig. 5, the top VB and bottom CB behaviors for both of these models are in qualitative agreement around $K$ valley edge, with the variation in the TB being wider for both geometric quantities. The significance of TW on these can be clearly observed together with the fact that $\mathrm{BC}$ toggles sign between VB and CB while this is not the case for the OMM.

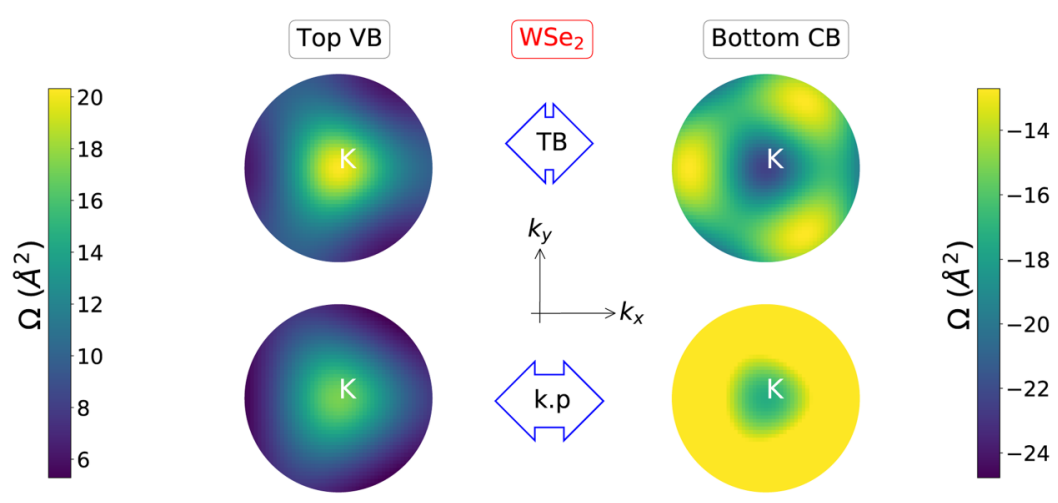

(a)

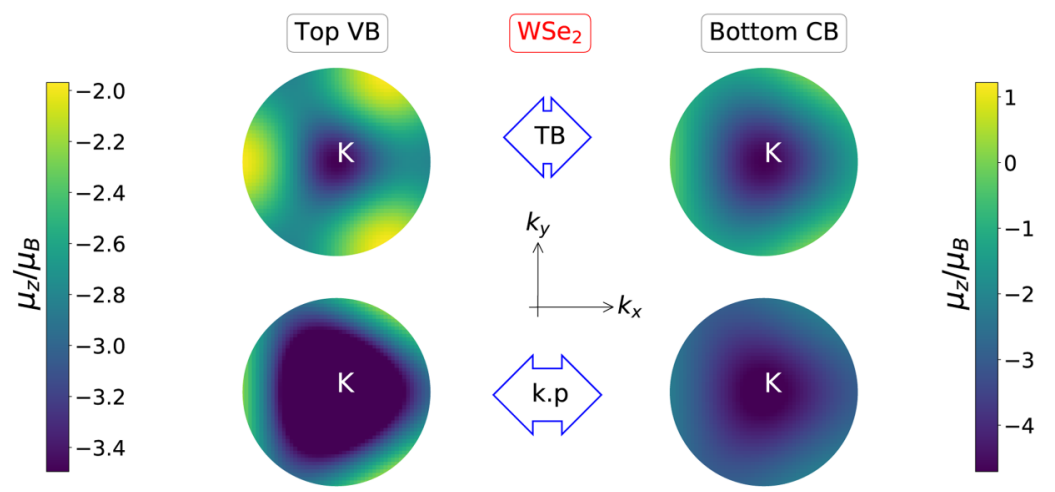

(b)
FIG. 5. (a) $B C$ and (b) OMM of (spin $\downarrow$ ) $C B$ and VB for an unstrained monolayer $\mathrm{WSe}_{2}$. The part of the Brillouin zone centered at the $K$ point over a radius of $0.12 \times$ $2 \pi / a$ is shown, maintaining the same axial orientation as in Fig. 2. 


\section{A. Effects of strain}

Figure 6 shows the effects of strain on the (a) BC and (b) $\mathrm{OMM}$ for the monolayer $\mathrm{WSe}_{2}$ over the $Q-K-M$ path within the Brillouin zone, where the $Q$ point lies exactly at midway between the $\Gamma$ and $K$ points. First considering the TB results, the geometric properties are seen to be inflated as the strain changes from compressive to tensile nature. However, this simple behavior is localized to the $K$ valley, especially for the VB. In the case of the $\mathrm{CB}$, the variation gets reversed beyond the halfway between the $Q-K$ panel, due to the satellite $C B$ valley at the $Q$ point. $^{25}$ Switching to $k \cdot p$ results, in the vicinity of the $K$ valley they display a behavior close to TB but again with somewhat reduced amplitudes. The incremental contribution of each term in the Hamiltonian [Eqs. (4)-(6)] indicates that the cubic term actually deteriorates the agreement with TB toward the $M$ point by introducing an extra curvature for both VB and $\mathrm{CB}$; yet it was observed in Fig. 3 to have a positive impact on the band structure for the same point.
These traits are more clearly demonstrated in Fig. 7 where the continuous tunability of both $\mathrm{BC}$ and $\mathrm{OMM}$ under hydrostatic strain $\varepsilon_{H}=\varepsilon_{x x}+\varepsilon_{y y}$ is displayed. Once again, $k \cdot p$ while in qualitative agreement with $\mathrm{TB}$ around the $K$ valley, it cannot reproduce the broad variations; particularly for the $\mathrm{CB}$ the $Q-K$ panel is not satisfactory. As a matter of fact, a separate $k \cdot p$ Hamiltonian needs to be invoked to replicate the correct behavior around the $\mathrm{CB} Q$ valley. ${ }^{25}$ Apart from these discrepancies at regions with relatively low curvature, both techniques reveal that the $K$ point geometric band properties can be doubled with respect to unstrained values by about $+5 \%$ hydrostatic strain.

We can offer a simple explanation for these increased geometrical band properties under tensile hydrostatic strain by making use of two-band electron-hole symmetric analytical expressions ${ }^{6,35}$ for the $K$ point: $\Omega_{z}= \pm 2\left(f_{2} a / E_{g}\right)^{2}$ and $\mu_{z}=\mu_{B} m_{0} / m^{*}$, where the strained bandgap $^{30} E_{g}=f_{1}+2 f_{4} \varepsilon_{H}$, and the strained effective mass ${ }^{30}$ $m^{*}= \pm \hbar^{2} E_{g} /\left[2\left(f_{2} a\right)^{2}\right]$. Since $f_{4}<0$ (cf. Table II), a tensile hydrostatic strain $\left(\varepsilon_{H}>0\right)$ decreases the $E_{g}$. Hence, this decrease in

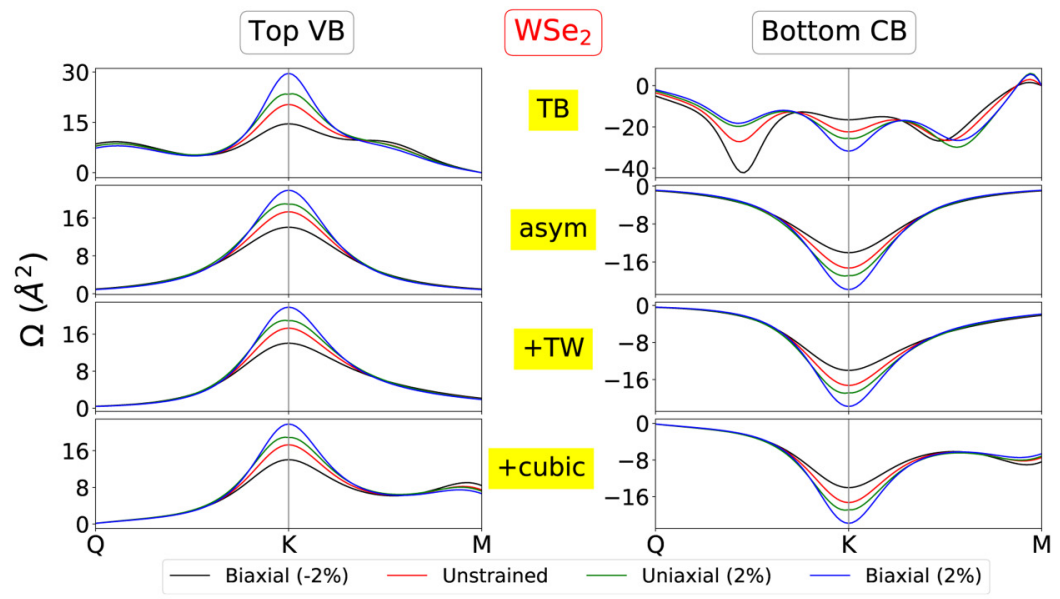

(a)

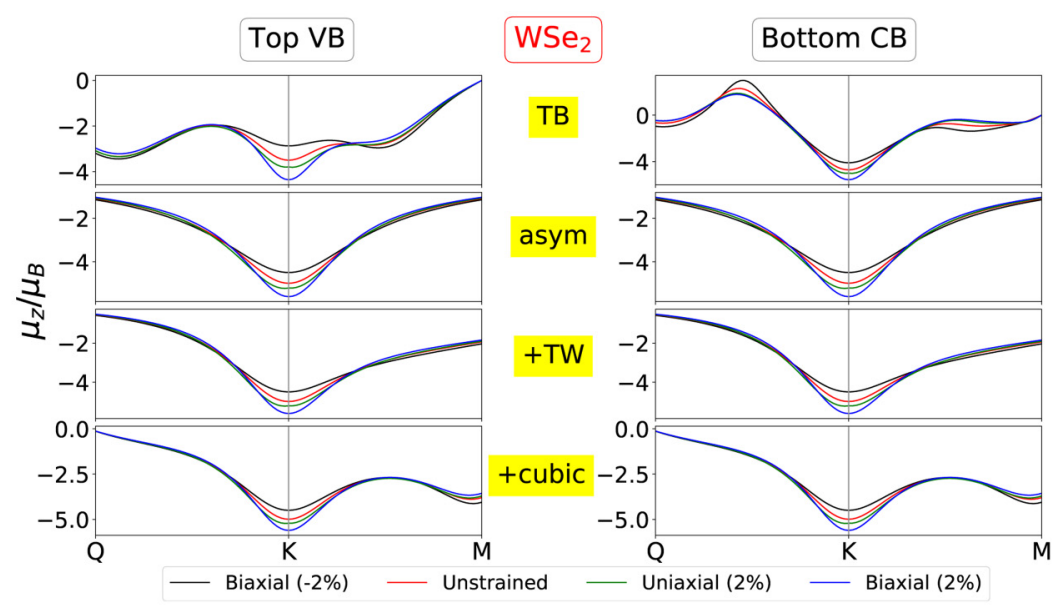

(b)
FIG. 6. Effect of strain for a monolayer $\mathrm{WSe}_{2}$ on (a) BC and (b) OMM of (spin $\downarrow$ ) CB and VB according to TB (top rows) and $k \cdot p$ (remaining rows) models, where for the latter the effect of each additional term [Eqs. (4)-(6)] is shown. 


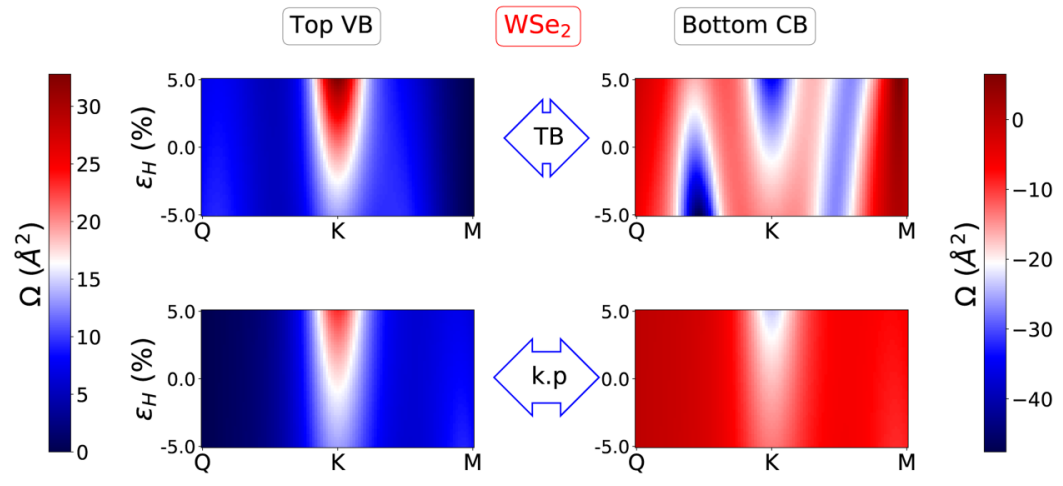

(a)

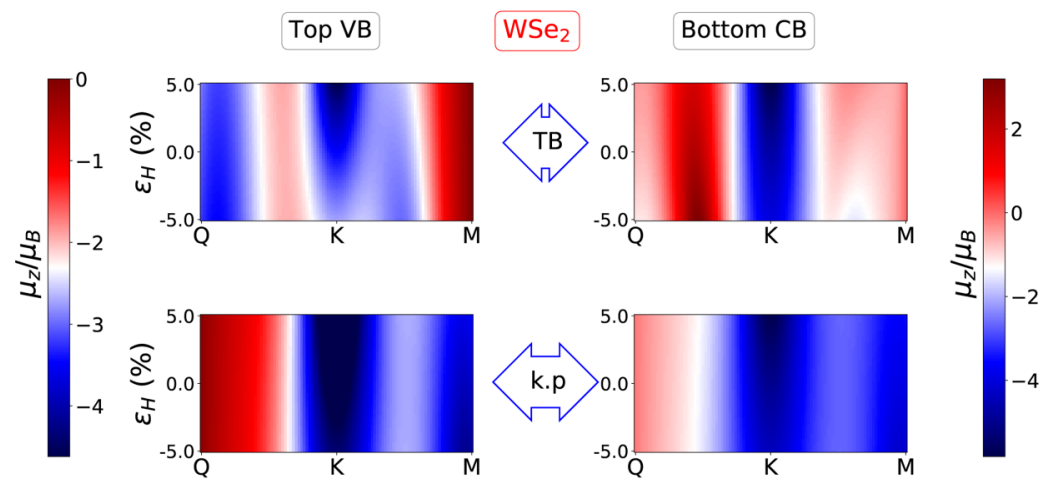

(b)

bandgap is the common origin for the improvement in both $\mathrm{BC}$ and OMM. As applying a tensile strain to a monolayer TMD is far less problematic than a compressive one which would lead to the buckling of the membrane, ${ }^{46}$ it warrants a realistic strain enhancement of the geometric band properties.

\section{CONCLUSIONS}

The appealing features of TMDs can be traced down largely to geometric band effects controlled by BC and OMM. Moreover, they can be widely tuned by exerting strain. To harness these in device applications, accurate and physically-transparent band structure tools are needed. In this work, we offer two options: a $k \cdot p$ model (a two- or a four-band) having an up-to-date parameter set and a strained extension of a three-band TB Hamiltonian. Despite their simplicity, both capture the essential physics that govern the variation of $\mathrm{BC}$ and $\mathrm{OMM}$, but with different validity ranges around the $K$ valley. Quantitatively, we report under reasonable biaxial tensile strains (about 2.5\%) that these can be doubled in value. It is straightforward to incorporate excitonic effects to this framework. $^{30}$ Thus, these models may serve for TMD device modeling purposes under electric, magnetic, or optical excitations in addition to strain. (2012). (2014).
FIG. 7. Effect of (compressive/tensile) hydrostatic strain $\varepsilon_{H}=\varepsilon_{x x}+\varepsilon_{y y}$ on (a) BC and (b) OMM of (spin $\downarrow$ ) CB and VB for a monolayer $\mathrm{WSe}_{2}$.

\section{REFERENCES}

${ }^{1}$ X. Xu, W. Yao, D. Xiao, and T. F. Heinz, Nat. Phys. 10, 343-350 (2014).

${ }^{2}$ S. Manzeli, D. Ovchinnikov, D. Pasquier, O. V. Yazyev, and A. Kis, Nat. Rev. Mater. 2, 17033 (2017).

${ }^{3}$ K. F. Mak, D. Xiao, and J. Shan, Nat. Photonics 12, 451-460 (2018).

${ }^{4} \mathrm{M}$. Yamamoto, Y. Shimazaki, I. V. Borzenets, and S. Tarucha, J. Phys. Soc. Jpn. 84, 121006 (2015).

${ }^{\mathbf{5}}$ J. R. Schaibley, H. Yu, G. Clark, P. Rivera, J. S. Ross, K. L. Seyler, W. Yao, and X. Xu, Nat. Rev. Mat. 1, 16055 (2016).

${ }^{6}$ D. Xiao, W. Yao, and Q. Niu, Phys. Rev. Lett. 99, 236809 (2007).

${ }^{7}$ W. Yao, D. Xiao, and Q. Niu, Phys. Rev. B 77, 235406 (2008).

${ }^{8}$ D. Xiao, G. B. Liu, W. Feng, X. Xu, and W. Yao, Phys. Rev. Lett. 108, 196802

${ }^{9}$ K. F. Mak, K. L. McGill, J. Park, and P. L. McEuen, Science 344, 1489-1492

${ }^{10}$ Z. Wu, B. T. Zhou, X. Cai, P. Cheung, G.-B. Liu, M. Huang, J. Lin, T. Han, L. An, Y. Wang, S. Xu, G. Long, C. Cheng, K. T. Law, F. Zhang, and N. Wang, Nat. Commun. 10, 611 (2019).

${ }^{11}$ T. Cao, G. Wang, W. Han, H. Ye, C. Zhu, J. Shi, Q. Niu, P. Tan, E. Wang, B. Liu, and J. Feng, Nat. Commun. 3, 887 (2012).

${ }^{12}$ D. V. Vanderbilt, Berry Phases in Electronic Structure Theory (Cambridge University Press, Cambridge, 2018).

${ }^{13}$ D. Xiao, M.-C. Chang, and Q. Niu, Rev. Mod. Phys. 82, 1959-2007 (2007).

${ }^{14}$ X. Zhang, W.-Y. Shan, and D. Xiao, Phys. Rev. Lett. 120, 077401 (2018).

${ }^{15}$ T. Cao, M. Wu, and S. G. Louie, Phys. Rev. Lett. 120, 077401 (2018).

${ }^{16}$ A. Srivastava and A. Imamoğlu, Phys. Rev. Lett. 115, 166802 (2015). 
17. Zhou, W.-Y. Shan, W. Yao, and D. Xiao, Phys. Rev. Lett. 115, 166803 (2015).

${ }^{18}$ M.-C. Chang and Q. Niu, J. Phys. Condens. Matter 20, 193202 (2008).

${ }^{19}$ M. Brooks and G. Burkard, Phys. Rev. B 95, 245411 (2017).

${ }^{20}$ D. V. Rybkovskiy, I. C. Gerber, and M. V. Durnev, Phys. Rev. B 95, 155406 (2017).

${ }^{21}$ G. Aivazian, Z. Gong, A. M. Jones, R. L. Chu, J. Yan, D. G. Mandrus, C. Zhang, D. Cobden, W. Yao, and X. Xu, Nat. Phys. 11, 148-152 (2015).

${ }^{22}$ A. Srivastava, M. Sidler, A. V. Allain, D. S. Lembke, A. Kis, and A. Imamoğlu, Nat. Phys. 11, 141-147 (2015).

${ }^{23}$ M. Schüler, U. D. Giovannini, H. Hübener, A. Rubio, M. A. Sentef, and P. Werner, e-print arXiv:1905.09404 (2019).

${ }^{24}$ S. Bertolazzi, J. Brivio, and A. Kis, ACS Nano 5, 9703-9709 (2011).

${ }^{25}$ A. Kormányos, G. Burkard, M. Gmitra, J. Fabian, V. Zólyomi, N. D. Drummond, and V. I. Fal'ko, 2D Mater. 2, 022001 (2015).

${ }^{\mathbf{2 6}}$ H. Rostami, R. Roldán, E. Cappelluti, R. Asgari, and F. Guinea, Phys. Rev. B 92, 195402 (2015)

${ }^{27}$ A. J. Pearce, E. Mariani, and G. Burkard, Phys. Rev. B 94, 155416 (2016).

${ }^{28}$ C. Sevik, J. R. Wallbank, O. Gülseren, F. M. Peeters, and D. Çakır, 2D Mater. 4, 035025 (2017).

${ }^{29}$ S. Fang, S. Carr, M. A. Cazalilla, and E. Kaxiras, Phys. Rev. B 98, 075106 (2018).

${ }^{30}$ S. Aas and C. Bulutay, Opt. Express 26, 28672-28681 (2018).

${ }^{31}$ Y. J. Zhang, T. Oka, R. Suzuki, J. T. Ye, and Y. Iwasa, Science 344, 725-728 (2014).

${ }^{32}$ Y. Li, J. Ludwig, T. Low, A. Chernikov, X. Cui, G. Arefe, Y. D. Kim, A. M. van der Zande, A. Rigosi, H. M. Hill, S. H. Kim, J. Hone, Z. Li,

D. Smirnov, and T. F. Heinz, Phys. Rev. Lett. 113, 266804 (2014).
${ }^{33}$ D. MacNeill, C. Heikes, K. F. Mak, Z. Anderson, A. Kormányos, V. Zólyomi, J. Park, and D. C. Ralph, Phys. Rev. Lett. 114, 037401 (2015).

${ }^{34}$ A. Kormányos, V. Zólyomi, N. D. Drummond, P. Rakyta, G. Burkard, and V. I. Fal'ko, Phys. Rev. B 88, 045416 (2013).

${ }^{35}$ S.-Y. Chen, Z. Lu, T. Goldstein, J. Tong, A. Chaves, J. Kunstmann, L. S. R. Cavalcante, T. Woźniak, G. Seifert, D. R. Reichman, T. Taniguchi, K. Watanabe, D. Smirnov, and J. Yan, Nano Lett. 19, 2464-2471 (2019).

${ }^{36}$ R. A. Bromley, R. B. Murray, and A. D. Yoffe, J. Phys. C Solid State Phys. 5, 759-778 (1972).

${ }^{37}$ E. Cappelluti, R. Roldán, A. Silva-Guillén, P. Ordejón, and F. Guinea, Phys. Rev. B 88, 075409 (2013).

${ }^{38}$ G. B. Liu, W. Y. Shan, Y. Yao, W. Yao, and D. Xiao, Phys. Rev. B 88, 085433 (2013).

${ }^{39}$ S. Fang, R. K. Defo, S. N. Shirodkar, S. Lieu, G. A. Tritsaris, and E. Kaxiras, Phys. Rev. B 92, 205108 (2018).

${ }^{40}$ J. Jeong, Y. H. Choi, K. Jeong, H. Park, D. Kim, and M. H. Cho, Phys. Rev. B 97, 075433 (2018).

${ }^{41}$ L. Zhang, Y. Huang, Q. Zhao, L. Zhu, Z. Yao, Y. Zhou, W. Du, and X. Xu, Phys. Rev. B 96, 155202 (2017).

${ }^{42}$ F. A. Rasmussen and K. S. Thygesen, J. Phys. Chem. C 119, 13169-83 (2015).

${ }^{43}$ K. S. Thygesen, 2D Mater. 4, 022004 (2017).

${ }^{44}$ W. Feng, Y. Yao, W. Zhu, J. Zhou, W. Yao, and D. Xiao, Phys. Rev. B 86, 165108 (2012).

${ }^{45}$ G. Wang, A. Chernikov, M. M. Glazov, T. F. Heinz, X. Marie, T. Amand, and B. Urbaszek, Rev. Mod. Phys. 90, 021001 (2018).

${ }^{46}$ B. Amorim, A. Cortijo, F. de Juan, A. Grushin, F. Guinea, A. Gutiérrez-Rubio, H. Ochoa, V. Parente, R. Roldán, P. San-Jose, J. Schiefele, M. Sturla, and M. Vozmediano, Phys. Rep. 617, 1-54 (2016). 